\title{
In Denial: Symptom Negation in New York City Emergency Department Chief Complaints
}

\author{
Jessica Sell* \\ Syndromic Surveillance, NYC DOHMH, Queens, NY, USA
}

\section{Objective}

To describe the effect of symptom negation in emergency department (ED) chief complaint data received by the New York City (NYC) Department of Health and Mental Hygiene (DOHMH), and to devise a solution to avoid syndrome and symptom misclassification for commonly used negations using SAS Perl Regular Expression (PRX) functions.

\section{Introduction}

In July 2016, 77\% of ED data was transmitted daily via Health Level 7 (HL7) messages, compared to only 27\% in July 2015 (Figure). During this same period, chief complaint (CC) word count has increased from an average of 3.8 words to 6.0 words, with a twenty-fold increase in the appearance of the word "denies" in the chief complaint (Figure). While HL7 messages provide robust chief complaint data, this may also introduce errors that could lead to symptom and syndrome misclassification.

\section{Methods}

Using SAS 9.4 and Tableau 9.3, we examined data submissions from 14 EDs responsible for $97 \%$ of the occurrences of the word 'denies' in chief complaints in July 2016.

To account for variation in chief complaint format among hospitals, we developed three PRX patterns to identify entire phrases in the chief complaint data field that began with conjugations of the word "deny" followed by various combinations of words, punctuation, spaces, and/or characters.

Pattern 1: '/DEN(Y|I(ES|ED $\mid \mathrm{NG}))(\backslash \mathrm{s}|| \mathrm{w}|(\backslash /)|(\backslash+)||,(\backslash \backslash))\{1$, $((\backslash).|(\backslash)|(\$)|(;)|(\backslash)) \mid(-)) /$ '

Pattern 2: ' '/DEN(Y|I(ES|ED $\mid \mathrm{NG}))(\backslash \mathrm{S}|\backslash \mathrm{W}|(\backslash /)|(\backslash+)|(\backslash \backslash))\{1$, $((\backslash).|(\backslash)|(\$)|(;)|(())|(-)|(),) /{ }^{\prime} ;$

Pattern 3: '/DENIES:( ||$w|\backslash|,)\{1\} / '$,$) ;$

We separated the 'denies' statement from the chief complaint and identified commonly negated symptoms. We then defined symptoms using keyword searches of the chief complaint and the 'denies' statement. We compared symptom classification with and without the consideration of symptom negation.

\section{Results}

Of the 14 EDs analyzed, we applied pattern 1 to 8 of the ED's, pattern 2 to $5 \mathrm{EDs}$, and patterns 2 and 3 to $1 \mathrm{ED}$. Approximately $98 \%$ of denies statements were extracted from chief complaints. Only $2 \%$ of symptom negation was not captured due to uncommon chief complaint format whose symptom negation didn't meet one of the previously described PRX patterns.

The most common words associated with a "denies" statement were: pain, chest, fever, loc, shortness, breath, vomiting, nausea, travel, headache, recent, trauma, history, abdominal, injury, diarrhea, SOB (shortness of breath), V (vomit), Head, N (nausea), PMH (past medical history), suicidal, dizziness, homicidal and D (diarrhea) (see Table).

By not taking negation into consideration in symptom definitions, between $3.5 \%$ and $16.5 \%$ of symptom visits were misclassified. Symptom misclassification varied greatly by hospital, ranging from $0 \%$ to $55 \%$.

\section{Conclusions}

As hospitals in NYC implement HL7 messaging, symptom negation is becoming increasingly common in chief complaint data. Current symptom definitions are based on keyword searches that do not take into account symptom negations. This leads to symptom misclassification, and could potentially cause false signals or inflate syndrome baselines, causing true signals to go undetected. SAS PRX functions can be used to flexibly identify symptom negation patterns and exclude them from syndrome definitions. Future studies will quantify the effect symptom negation has had on signal frequency in NYC, and examine symptoms associated with other forms of negation such as "Personal Medical History", "No" and "Negative."

Most Common Symptoms Denied in Emergency Department Chief Complaints

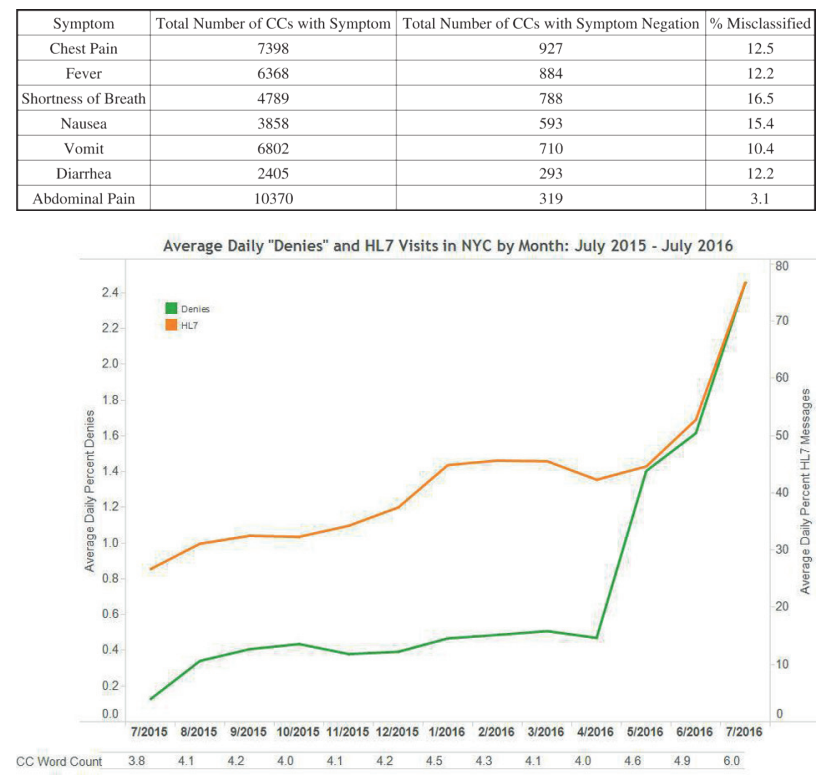

Keywords

Symptom Negation; Perl Regular Expressions; HL7 Data

*Jessica Sell

E-mail: jsell@health.nyc.gov 